Article

\title{
Antimicrobial Resistance and Molecular Epidemiology of Corynebacterium striatum Isolated in a Tertiary Hospital in Turkey
}

\author{
Nergis Asgin ${ }^{1, *}$ and Baris Otlu ${ }^{2}$ \\ 1 Department of Medical Microbiology, Faculty of Medicine, Karabuk University, 78100 Karabuk, Turkey \\ 2 Department of Medical Microbiology, Faculty of Medicine, Inonu University, 44280 Malatya, Turkey; \\ baris.otlu@inonu.edu.tr \\ * Correspondence: nergisasgin@karabuk.edu.tr; Tel.: +90-370-4189446
}

Received: 16 January 2020; Accepted: 18 February 2020; Published: 19 February 2020

\begin{abstract}
Although Corynebacterium striatum is part of the human flora, it has recently drawn attention both for its multidrug resistance and its role as an invasive infection/outbreak agent. This cross-sectional study aimed to determine the antimicrobial resistance and clonal relationships among C. striatum strains. In total, 81 C. striatum strains were identified using Phoenix $-100^{\mathrm{TM}}$ (BD, Sparks, MD, USA). The antimicrobial resistance of the strains was determined using the Kirby-Bauer disk diffusion method. Clonal relatedness among the strains was performed via arbitrarily primed polymerase chain reaction (AP-PCR). All 81 C. striatum strains were resistant to penicillin, cefotaxime, ciprofloxacin, and tetracycline, but susceptible to vancomycin and linezolid. The resistance rates to gentamicin, erythromycin, and clindamycin were $34.6 \%, 79 \%$, and $87.7 \%$ respectively. AP-PCR results showed no predominant clone among the $C$. striatum strains. Corynebacterium striatum is reportedly the cause of an increasing number of invasive infections/outbreaks. Moreover, treatment options are limited. The study showed that vancomycin, linezolid, and gentamicin can be selected for the empirical treatment of $C$. striatum infections. Although no single-clone outbreak was observed in our hospital, small clonal circulations were observed within some units, indicating cross-contamination. Therefore, a comprehensive infection control program is warranted in future.
\end{abstract}

Keywords: antimicrobial resistance; arbitrarily primed polymerase chain reaction; clone; Corynebacterium striatum; molecular epidemiology, nosocomial outbreak

\section{Introduction}

The Corynebacterium species (spp) are aerobic, non-spore-forming, club-shaped Gram-positive rods. They are ubiquitous in the environment (soil and water) and some species are commensal of normal human skin and mucous membranes. To date, over 100 species have been identified, and 54 of them are associated with human infections [1,2]. Previously, only Corynebacterium diphtheriae was considered an infectious agent, whereas other Corynebacterium spp. were traditionally dismissed as contaminant bacteria when isolated from clinical specimens. The prevalence of diphtheria has decreased globally after effective vaccination programs. Recently, various non-diphtheriae Corynebacterium spp. have been increasingly reported as infectious agents in patients with long-term hospitalization, invasive intervention, and underlying diseases [2,3]. All medically relevant species in the Corynebacterium genus are catalase positive and nonmotile. They are divided into lipophilic and nonlipophilic as well as fermentative and nonfermentative. Most of the Corynebacterium spp. can be isolated from $5 \%$ sheep blood agar. Lipophilic species have enhanced growth in the presence of lipids such as Tween-80. Fermentative species produce acid from various sugars, including glucose, maltose, 
and sucrose. The most frequently isolated species from clinical samples of humans are C. jeikeium, C. striatum, C. urealyticum, and C. amycolatum [1,4]. C. jeikeium and C. urealyticum are lipophilic and nonfermentative, whereas C. striatum and C. amycolatum are nonlipophilic and fermentative [1]. Corynebacterium jeikeium is frequently isolated from clinical specimens and has demonstrated nosocomial transmission. They can account for a wide range of human infections, such as respiratory tract infections, septic arthritis, and endocarditis, especially among immunosuppressive patients, including patients with neutropenia, malignancy, and AIDS [2,3]. Corynebacterium striatum is the second most commonly isolated Corynebacterium species after C. jeikeium [5]. Colonies are convex, shiny, moist, and creamy on the medium. The strains produce acid from glucose and reduce nitrate, whereas the Christie-Atkinson-Munch-Peterson (CAMP) test is variable [2].

Corynebacterium striatum was first reported in 1980 as the cause of a pleuropulmonary infection in a patient with chronic lymphocytic leukemia [6]. In 1993, the first nosocomial outbreak caused by C. striatum was reported, proving that the infection was transmitted from patient to patient via the hands of the hospital staff [7]. Severe invasive infections such as meningitis, blood-stream infections, endocarditis, and osteomyelitis caused by $C$. striatum have been reported in both immunosuppressive and immunocompetent individuals worldwide [8-11]. Additionally, nosocomial outbreaks due to C. striatum have been reported in several countries $[12,13]$. Moreover, the increase in antimicrobial resistance of $C$. striatum is a great concern. Previously, it was susceptible to many drugs including penicillin, but it has recently demonstrated high-level resistance to antibiotics such as beta-lactams, macrolides, aminoglycosides, and quinolones [14-16]. Furthermore, there are some reports on the C. striatum strains resistant to daptomycin, which is considered the last-line drug [17,18]. In Turkey, limited data are available on antimicrobial susceptibilities of $C$. striatum, and no study has reported the molecular epidemiology of $C$. striatum. Therefore, we aimed to determine antimicrobial resistance profiles and molecular epidemiology of $C$. striatum isolated from inpatients at a tertiary hospital in Turkey.

\section{Results}

\subsection{Demographic and Clinical Characteristics of Patients}

The demographic and clinical characteristics of the patients from whom strains were obtained from the hospital information system are presented in Table 1.

Table 1. Demographic and clinical features of patients $(n=81)$

\begin{tabular}{cc}
\hline Data & Number (\%) \\
\hline Age (years) & $12(14.8)$ \\
$40-59$ & $42(51.9)$ \\
$60-79$ & $27(33.3)$ \\
$\geq 80$ & \\
Gender & $39(48.1)$ \\
Female & $42(51.9)$ \\
Male & $68(84.0)$ \\
Hospital Unit & $23(28.4)$ \\
Intensive Care Unit (ICU) & $22(27.2)$ \\
Surgical ICU & $16(19.8)$ \\
Internal medicine ICU & $7(8.6)$ \\
Reanimation ICU & $13(16.0)$ \\
Coronary ICU & $5(6.2)$ \\
Wards & $4(4.9)$ \\
General surgery & $4(4.9)$ \\
Palliative care & \\
Respiratory &
\end{tabular}


Table 1. Cont.

\begin{tabular}{cc}
\hline Data & Number (\%) \\
\hline Specimens & $35(43.2)$ \\
Blood & $24(29.6)$ \\
Endotracheal aspirate & $11(13.6)$ \\
Wound & $7(8.7)$ \\
Sputum & $4(4.9)$ \\
Bronchoalveolar lavage fluid & $18(22.2)$ \\
Underlying Diseases & $14(17.3)$ \\
${ }^{*}$ COPD & $12(14.8)$ \\
Heart failure/ attack & $9(11.1)$ \\
Cerebrovascular disease & $9(11.1)$ \\
Cardiac arrest & $8(9.9)$ \\
Renal failure & $7(8.6)$ \\
Acute abdomen with ileus & $5(6.2)$ \\
Surgical site infection & $4(4.9)$ \\
Sepsis & $4(4.9)$ \\
Gastrointestinal hemorrhage & $3(3.7)$ \\
Traumatic pneumothorax & $3(3.7)$ \\
Femur fracture &
\end{tabular}

The mean age of the patients was $71.7 \pm 11.7$ years (range, $40-95$ years) and $76.5 \%$ of the patients were aged $\geq 65$ years. Of the 81 patients, 42 (51.8\%) were male, and $39(48.2 \%)$ were female. The mean age of the male and female was similar and was $69.9 \pm 11.4$ and $73.6 \pm 11.9$, respectively $(P=0.165)$. The distributions of patients according to age groups and genders are presented in Table S1. No statistically significant difference was observed between age groups and genders of patients $(P=0.446)$.

Of 81 patients, $68(84 \%)$ patients received treatment in the intensive care unit (ICU), whereas $13(16 \%)$ were treated in the hospital wards. The mean age of patients in the ICU was similar to those in the wards and was $72.7 \pm 11.5$ and $67.2 \pm 11.6$, respectively $(P=0.134)$.

All patients had at least one underlying disease. The most common underlying diseases were chronic obstructive pulmonary disease (COPD) $(22.2 \%)$, heart failure/attack $(17.3 \%)$, and cerebrovascular disease $(14.8 \%)$.

Of the 81 C. striatum strains, $68(84 \%)$ were isolated from ICUs, and $13(16 \%)$ were isolated from various wards. The strains obtained $35(43.2 \%)$ from blood, 35 (43.2\%) from respiratory samples (endotracheal aspirate, sputum, bronchoalveolar lavage fluid), and $11(13.6 \%)$ from wounds.

\subsection{Antibiotic Resistance Profiles of Isolates}

All of the strains $(100 \%)$ were resistant to penicillin, cefotaxime, ciprofloxacin, and tetracycline, but susceptible to vancomycin and linezolid. The rates of resistance to gentamicin, erythromycin, and clindamycin were $34.6 \%, 79 \%$, and $87.7 \%$, respectively. The antibiotic resistance rates of strains isolated from ICUs and wards are shown in Table 2. The resistance rates of erythromycin, gentamicin, and clindamycin were similar in the strains isolated from ICUs and wards. 
Table 2. Antibiotic resistance rates of Corynebacterium striatum strains isolated from the ICUs and wards.

\begin{tabular}{ccccc}
\hline & Total $\mathbf{( n = 8 1 )}$ & ICUs $(\mathbf{n}=\mathbf{6 8})$ & Wards $\mathbf{( n = 1 3 )}$ & $P$-value \\
\hline Antibiotic & $\mathrm{n}(\%)$ & $\mathrm{n}(\%)$ & $\mathrm{n}(\%)$ & \\
Erythromycin & $64(79)$ & $55(80.8)$ & $9(69.2)$ & 0.363 \\
Clindamycin & $71(87.7)$ & $59(86.6)$ & $12(92.3)$ & 0.578 \\
Gentamicin & $28(34.6)$ & $23(33.8)$ & $5(38.5)$ & 0.747 \\
Ciprofloxacin & $81(100)$ & $68(100)$ & $13(100)$ & $\mathrm{Na}$ \\
Penicillin & $81(100)$ & $68(100)$ & $13(100)$ & $\mathrm{Na}$ \\
Cefotaxime & $81(100)$ & $68(100)$ & $13(100)$ & $\mathrm{Na}$ \\
Ciprofloxacin & $81(100)$ & $68(100)$ & $13(100)$ & $\mathrm{Na}$ \\
Tetracycline & $81(100)$ & $68(100)$ & $13(100)$ & $\mathrm{Na}$ \\
Vancomycin & $0(0)$ & $0(0)$ & $0(0)$ & $\mathrm{Na}$ \\
Linezolid & $0(0)$ & $0(0)$ & $0(0)$ & $\mathrm{Na}$ \\
\hline
\end{tabular}

Abbreviations: ICU: Intensive care unit; Na: Not applicable.

\subsection{AP-PCR Results}

The dendrogram generated according to the AP-PCR profiles of the C. striatum strains is presented in Figure 1. AP-PCR results showed that there was no predominant clone among the C. striatum strains. The 81 C. striatum strains showed 54 different profiles, and 36 of the 81 strains were clonally related and formed nine clusters (tolerance: 1.0; cutoff: 95\%). Thus, the clustering rate of the strains was $44.4 \%$ $(36 / 81)$.

The distribution of genotyped and sporadic strains according to the ICU and ward is shown in Table S2. No statistically significant difference was observed between the ICU and ward with respect to genotyped/sporadic strains $(P=0.120)$.

The distribution of genotyped and sporadic strains according to age groups of patients (40-59, $60-79$, and $>80$ years) is shown in Table S3. No statistically significant difference was observed between age groups with respect to genotyped/sporadic strains $(P=0.091)$.

The clusters were named with lower case letters: $a, b, c, d, e, f, g, h$, and $k$. The largest cluster was genotype a, which was composed of eight strains and had $10 \%$ of the strains. Genotypes $\mathrm{k}$ had five strains, and genotypes b, c, d, and h had four strains each. Genotypes e had three strains. The smallest clusters were genotypes $\mathrm{f}$ and $\mathrm{g}$, each with two strains.

The characteristics of the 36 C. striatum strains genotyped using the AP-PCR method are shown in Table 3. 


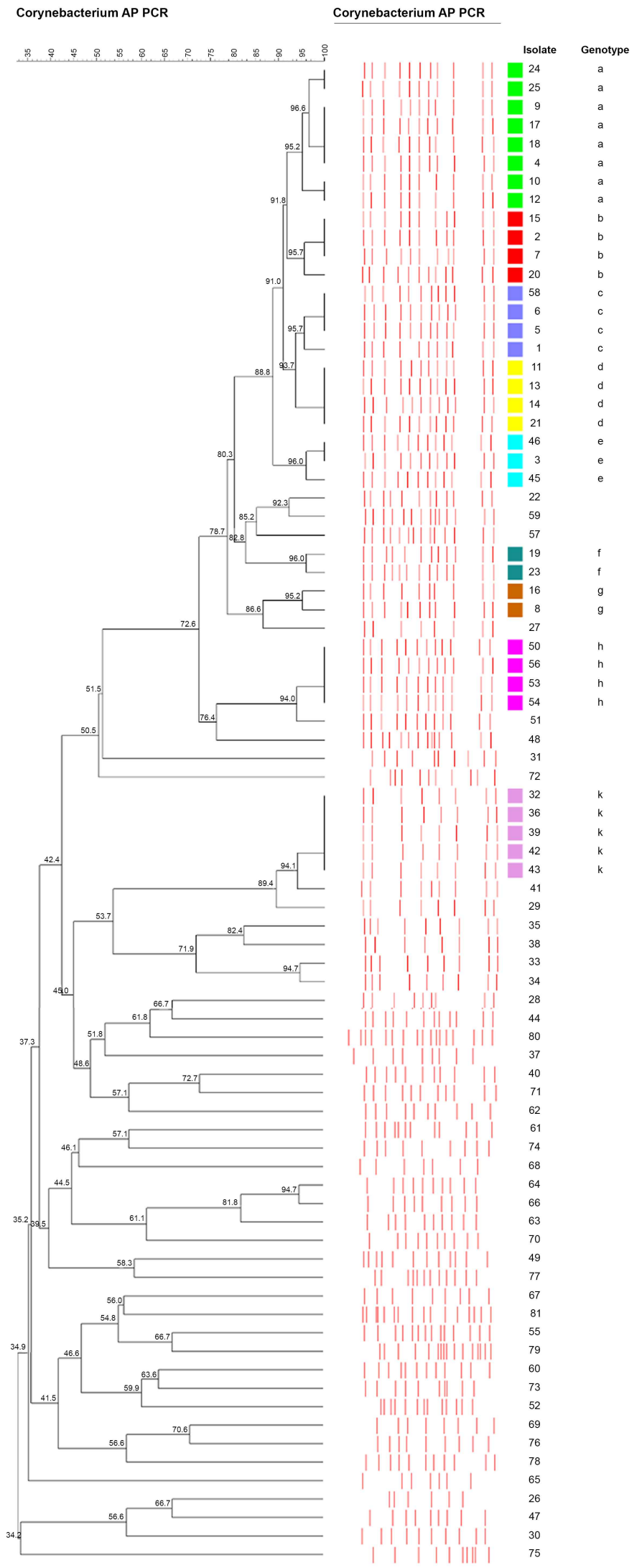

Figure 1. The dendrogram generated according to arbitrarily primed polymerase chain reaction profiles of Corynebacterium striatum strains. The clusters were named with lowercase letters: a, b, c, d, e, f, g, h, and $\mathrm{k}$. 
Table 3. Characteristics of Corynebacterium striatum strains genotyped by arbitrarily primed polymerase chain reaction $(n=36)$.

\begin{tabular}{|c|c|c|c|c|c|}
\hline Strain no & Department & Source & Isolation Date & $\begin{array}{c}\text { Antibiotic } \\
\text { Susceptibility }\end{array}$ & Genotype \\
\hline 4 & surgical ICU & EA & 13.4.2015 & Va, Lzd & a \\
\hline 9 & internal medicine ICU & EA & 18.4 .2015 & Va, Lzd, Gn, E & a \\
\hline 10 & reanimation ICU & EA & 8.2 .2016 & $\mathrm{Va}, \mathrm{Lzd}$ & a \\
\hline 12 & surgical ICU & Blood & 2.10 .2015 & Va, Lzd & a \\
\hline 17 & internal medicine ICU & EA & 4.10 .2015 & Va, Lzd, Gn, E, Da & a \\
\hline 18 & palliative care unit & wound & 27.12.2015 & Va, Lzd, E & a \\
\hline 24 & coronary ICU & sputum & 21.11.2015 & Va, Lzd & a \\
\hline 25 & surgical ICU & wound & 7.2.2015 & Va, Lzd & a \\
\hline 2 & surgical ICU & Blood & 8.4 .2015 & Va, Lzd, Gn & $\mathrm{b}$ \\
\hline 7 & internal medicine ICU & EA & 19.10.2015 & Va, Lzd, Gn & $\mathrm{b}$ \\
\hline 15 & reanimation ICU & EA & 10.1.2015 & Va, Lzd, Gn & $\mathrm{b}$ \\
\hline 20 & palliative care unit & sputum & 13.12.2015 & $\mathrm{Va}, \mathrm{Lzd}, \mathrm{Gn}$ & $\mathrm{b}$ \\
\hline 58 & internal medicine ICU & BLF & 11.5.2015 & Va, Lzd, Gn & c \\
\hline 1 & surgical ICU & Blood & 30.1 .2015 & Va, Lzd, Gn & c \\
\hline 5 & reanimation ICU & EA & 7.2.2015 & Va, Lzd, Gn & c \\
\hline 6 & internal medicine ICU & Blood & 29.5.2015 & Va, Lzd, E & c \\
\hline 11 & internal medicine ICU & Blood & 5.5.2015 & Va, Lzd, Gn & $\mathrm{d}$ \\
\hline 13 & surgical ICU & EA & 23.10.2015 & Va, Lzd, Gn & $\mathrm{d}$ \\
\hline 14 & internal medicine ICU & BLF & 26.8.2015 & Va, Lzd & $\mathrm{d}$ \\
\hline 21 & surgical ICU & EA & 14.1.2016 & Va, Lzd & $\mathrm{d}$ \\
\hline 3 & internal medicine ICU & EA & 4.1.2016 & $\mathrm{Va}, \mathrm{Lzd}$ & e \\
\hline 45 & internal medicine ICU & sputum & 6.11 .2015 & Va, Lzd & e \\
\hline 46 & internal medicine ICU & EA & 4.12 .2015 & Va, Lzd & e \\
\hline 19 & surgical ICU & wound & 17.4.2015 & Va, Lzd, Gn & $\mathrm{f}$ \\
\hline 23 & surgical ICU & sputum & 4.2 .2015 & Va, Lzd, Gn & $\mathrm{f}$ \\
\hline 8 & internal medicine ICU & BLF & 3.4 .2015 & Va, Lzd, Gn & $g$ \\
\hline 16 & internal medicine ICU & Blood & 24.3.2015 & Va, Lzd, Gn & $\mathrm{g}$ \\
\hline 50 & surgical ICU & Blood & 24.7.2015 & Va, Lzd, Gn & $\mathrm{h}$ \\
\hline 53 & surgical ICU & Blood & 1.4.2015 & Va, Lzd, Gn & $\mathrm{h}$ \\
\hline 54 & coronary ICU & EA & 8.1.2015 & $\mathrm{Va}, \mathrm{Lzd}, \mathrm{Gn}, \mathrm{E}, \mathrm{Da}$ & $\mathrm{h}$ \\
\hline 56 & coronary ICU & sputum & 24.2 .2015 & $\mathrm{Va}, \mathrm{Lzd}, \mathrm{Gn}$ & $\mathrm{h}$ \\
\hline 32 & surgical ICU & EA & 21.12.2015 & Va, Lzd & $\mathrm{k}$ \\
\hline 36 & reanimation ICU & Blood & 23.5.2015 & Va, Lzd, Gn, E, Da & $\mathrm{k}$ \\
\hline 39 & reanimation ICU & EA & 4.7.2015 & $\mathrm{Va}, \mathrm{Lzd}$ & $\mathrm{k}$ \\
\hline 42 & surgical ICU & EA & 20.5.2015 & Va, Lzd, Gn & $\mathrm{k}$ \\
\hline 43 & reanimation ICU & BLF & 23.11.2015 & Va, Lzd, Gn, E & $\mathrm{k}$ \\
\hline
\end{tabular}

Abbreviations: ICU: Intensive care unit, EA: Endotracheal aspirate, BLF: Bronchoalveolar lavage fluid. Va: Vancomycin, Lzd: Linezolid, Gn: Gentamicin, E: Erythromycin, Da: Clindamycin.

All 36 genotyped strains were obtained from ICUs and palliative care units. The strains in genotype a $(n=8)$ were isolated from different ICUs and palliative care units over one year and did not show unique antibiotic resistance profiles. The four strains in genotype $b$ strains were isolated on different dates from various units. They had the same resistance profile and were susceptible to vancomycin, linezolid, and gentamicin. The three strains in genotype e were isolated from respiratory samples obtained from internal medicine ICU over a period of two months. The antibiotic resistance profiles of the strains were the same and were only susceptible to vancomycin and linezolid. The two strains each in genotypes $f$ and $g$ were obtained from the surgical ICU over a period of two months and internal medicine ICU over a period of 10 days, respectively, and were susceptible to vancomycin, linezolid, and gentamicin. 


\section{Discussion}

Corynebacterium striatum is frequently isolated from patients hospitalized long-term, treated with invasive procedures, and having underlying diseases such as COPD $[12,13,19,20]$. In this study, the most common underlying disease in patients was COPD, followed by cardiovascular diseases and cerebrovascular diseases. Additionally, the increasing drug resistance in C. striatum strains limits treatment options. In earlier reports, C. striatum strains were susceptible to many antibiotics [21,22]. However, they have been reported to be multidrug-resistant in recent studies [16,23-25]. In the present study, all the C. striatum strains were resistant to penicillin, cefotaxime, and ciprofloxacin. In C. striatum strains, the bla gene that encodes class A beta-lactamase is responsible for penicillin resistance, whereas the amp $C$ gene that encodes class $C$ beta-lactamase is responsible for cefotaxime resistance [23]. Quinolone resistance was found to be associated with point mutations in the gyrase subunit A structural gene region $[9,26]$. In addition, it was reported that antibiotic-resistant mutant strains emerged in bacteria such as Corynebacterium spp., which are found in normal flora, following the use of quinolone [23]. A study conducted by Mumcuoglu et al. on 746 C. striatum strains isolated in Turkey between 2010 and 2014 demonstrated that the rates of resistance to penicillin and ciprofloxacin were $80 \%$ and $83 \%$, respectively [27]. Similarly, Neemuchwala et al. reported high resistance rates (99.9\% to penicillin and $95.4 \%$ to ciprofloxacin) in 931 C. striatum strains isolated in Canada between 2011 and 2016 [28]. Conversely, Alibi et al. [23] from Tunisia reported lower resistance rates ( $82.5 \%$ to penicillin and $36.5 \%$ to ciprofloxacin) in 63 C. striatum strains isolated between 2011 and 2014. These results may be due to differences in the regional antibiotic resistance profiles of the strains, as well as the different collection dates. Aminoglycoside resistance has been gradually increasing in C. striatum strains. The most common mechanism has been reported to be the enzymatic inactivation of the antibiotic molecule [23]. In this study, the resistance to gentamicin was 34.6\%, whereas Mumcuoglu et al. [27] reported it to be $17.6 \%$, Neemuchwala et al. [28] as $7.2 \%$, and Alibi et al. [23] as 6.3\%. However, Mumcuoglu et al. reported that all 231 (24.8\%) strains isolated in 2014 were multidrug-resistant and also resistant to gentamicin [27]. Alibi et al. observed multidrug resistance in $31(49.2 \%)$ of 63 C. striatum strains [23]. Wang et al. from China reported that $183(95.3 \%)$ of 192 C. striatum strains isolated between 2017 and 2018 were multidrug-resistant and gentamicin resistance rate was $22.9 \%(44 / 192)$ [24]. In the present study, all 81 strains $(100 \%)$ were resistant to at least three antibiotic groups.

We have found high resistance rates to erythromycin and clindamycin: 79\% (64/81) and 87.7\% (71/81), respectively. Similarly, Ortiz-Pérez et al. reported resistance rates of $76.4 \%$ to erythromycin and $74 \%$ to clindamycin in 256 C. striatum strains [29]. Resistance to macrolide and lincosamide was reported to be the result of a modification in the region where the antibiotics bind to the methylase enzyme, which is mostly encoded by erm $(X)$ genes [26-30]. In this study, all strains $(100 \%)$ were resistant to tetracycline. Barberis et al. [30] reported tetracycline resistance of $20 \%(11 / 55)$, whereas Wang et al. [24] and Mc Mullen et al [15] reported as 58.3\% (112/192) and 62\% (128/206) respectively. It has also been reported that the tet $A$ and $\operatorname{tet} B$ genes play a role in the tetracycline resistance of $C$. striatum strains and that the mechanism of resistance involves an active efflux pump [26,31]. Many recent studies have not reported resistance to vancomycin and linezolid [16,23-25]. Similarly, in our study, all strains were susceptible to vancomycin and linezolid.

Recently, molecular genotyping methods have been widely used to obtain knowledge about the origin, reservoirs, and circulation patterns of pathogens in case of nosocomial outbreak suspicion. PCR-based molecular typing methods, such as AP-PCR and repetitive element palindromic-PCR (rep-PCR), are faster, easier to use, and easier to interpret than pulsed-field gel electrophoresis (PFGE) which is the gold standard method. Although there is some variability depending on the type of microorganism studied, PCR-based molecular typing methods are reported to have lower discrimination power and reproducibility than PFGE, particularly among closely related strains [32]. In this study, we used the AP-PCR method for molecular epidemiological analysis of the C. striatum strains. A total of $36(44.4 \%)$ of 81 strains were clonally related, and nine clusters were formed. 
As the majority of the clonally related strains were obtained from the surgical and internal medicine ICUs, it was understood that these units were the main sources. The results of AP-PCR revealed no single-clone outbreak at our hospital, but multiclonal spread had taken place.

Similarly, Mc Mullen et al. [15] reported multiclonal spread among 85 C. striatum strains using rep-PCR, isolated between 2013 and 2015 in USA. Although half of the strains were in one group, the remaining strains were distributed into 11 different groups, and the strains in the dominant clone had different antibiotic resistance profiles. Furthermore, they reported that this clonal diversity may be due to the isolation of strains in the non-outbreak period. Alibi et al. [23] detected 22 PFGE profile in 63 C. striatum strains from Tunisia and reported high clonal diversity among strains. Wang et al. [19] reported that three epidemic clones were spread by PFGE in 82 C. striatum strains isolated from patients' respiratory samples between 2013 and 2014 in China. Wang et al. [24] applied PFGE to 192 C. striatum isolated between 2017 and 2018, and also performed whole genome sequencing in 91 C. striatum isolates and reported that there was multiclonal spread within the hospital simultaneously.

In contrary, there are studies reporting single clonal spread in the literature. Baio et al. [33] in 2013 from Brazil and Campanila et al. [31] in 2009 from Italy reported single clonal spread using the PFGE method. Verroken et al. performed both rep-PCR and PFGE methods in 10 outbreak C. striatum strains in 2011 in Belgium [13]. Only a single clone was detected by rep-PCR, whereas three distinct types were detected using PFGE. Therefore, they emphasized the high discriminative power of PFGE.

According to our study results, although there was no single-clone outbreak in our hospital, small clonal circulations were observed within some units. For instance, in small groups, such as genotypes e, $\mathrm{f}$, and $\mathrm{g}$, strains were isolated from the same unit and had the same antibiotic resistance profile and were obtained over a short period. These results indicate cross-contamination. Therefore, we should strictly enforce contact precautions. Hand hygiene is a simple and powerful infection prevention method. Monitoring the use of alcohol-based hand rubs of healthcare workers (HCWs) before and after contact with the patient and encouraging HCWs to remain bare below the elbows are easy and cost-effective measures that can be taken [34,35]. In addition, environmental hygiene should be enhanced. Hospital surfaces and medical equipment should be cleaned with approved disinfectants at appropriate concentration and times.

To the best of our knowledge, this is the first report on the molecular epidemiological analysis of C. striatum clinical isolates in Turkey. Of course, there are some limitations. First, this was a retrospective and monocenter study. Second, the $C$. striatum strains were obtained during routine laboratory work and were not accompanied by clinical data, antibiotic treatment regimens, or whether the strains were colonizers or causes of infection. Third, the AP-PCR method was used for molecular epidemiological analysis of the isolates instead of the PFGE. PFGE is a labor-intensive method that requires experienced staff and special equipment. AP-PCR is a more practical method because it is easy and cheap to perform and obtained the results within a short time. Therefore, AP-PCR can be performed during an outbreak, allowing the source of the outbreak to be rapidly detected.

\section{Materials and Methods}

\subsection{Study Design}

This cross-sectional study was conducted between November 2018-February 2019 at the Karabuk University Training and Research Hospital, a 440-bed tertiary hospital in the city of Karabuk, western Black Sea region of Turkey.

\subsection{Isolation and Identification of C. striatum Strains}

The study included 81 C. striatum strains isolated from routine clinical samples of inpatients at Karabuk University Training and Research Hospital between January 2015 and February 2016. 
The strains were stored in brain-hearth infusion broth with $20 \%$ glycerol at $-80{ }^{\circ} \mathrm{C}$ until use. Only one strain from each patient was included. Repetitive, other Corynebacterium species, contaminated, or unanimated strains were excluded.

Clinical samples (blood, wound, endotracheal aspirate, bronchoalveolar lavage fluid, sputum) collected from inpatients were inoculated on 5\% sheep blood Columbia agar [Becton Dickinson and Company (BD), Sparks, MD, USA], eosin methylene blue (EMB) agar (BD), and chocolate agar (BD). For blood culture, $8-10 \mathrm{~mL}$ of each patient's blood was inoculated into BD BACTEC Plus vials and incubated in the Bactec FX 40 (BD, MD, USA) automated blood culture system for seven days. Samples with positive signals were inoculated on $5 \%$ sheep blood Columbia agar, EMB agar, and chocolate agar plates. After an incubation period of $24-48 \mathrm{~h}, 35^{\circ} \mathrm{C}$ in $5 \% \mathrm{CO}_{2}$ atmosphere conditions, Gram staining was performed on the catalase-positive colonies and microscopically examined. When Gram-positive pleomorphic bacilli were seen, the colonies were identified using the BD Phoenix ${ }^{\mathrm{TM}}$ automated system. Identification of isolates was confirmed using the matrix-assisted laser desorption/ionization time-of-flight (MALDI-TOF) method with the VITEK@-MS device (BioMérieux, Marcy-l'Étoile, France) at the Inonu University School of Medicine, Molecular Microbiology Laboratory in Malatya.

\subsection{Antibiotic Susceptibility Testing}

The antibiotic susceptibility of the strains was determined using the Kirby-Bauer disk diffusion method. The bacterial suspension adjusted $0.5 \mathrm{Mc}$ Farland turbidity was inoculated by sterile cotton swap to the surface of Mueller-Hinton fastidious agar (MH-F, BD, MD, USA) plate containing 5\% defibrinated horse blood and $20 \mathrm{mg} / \mathrm{L} \beta$-NAD. Nine antibiotic discs were used: Penicillin (1 unit), ciprofloxacin $(5 \mu \mathrm{g})$, gentamicin $(10 \mu \mathrm{g})$, vancomycin $(5 \mu \mathrm{g})$, linezolid $(10 \mu \mathrm{g})$, erythromycin $(15 \mu \mathrm{g})$, clindamycin $(2 \mu \mathrm{g})$, cefotaxime $(5 \mu \mathrm{g})$, and tetracycline $(30 \mu \mathrm{g})$. Antibiotic discs were provided by BBL (BD, Sparks MD, USA) and MAST (MAST Group, Liverpool, UK). The antibiotic discs were placed on the MH-F agar plates within $15 \mathrm{~min}$ of inoculation on the plates. The plates were incubated for $16-24 \mathrm{~h}$ at $35^{\circ} \mathrm{C}$ in $5 \% \mathrm{CO}_{2}$ atmosphere conditions. The inhibition zone diameters formed around the antibiotic discs were measured and interpreted using the European Committee on Antimicrobial Susceptibility Testing (EUCAST) guidelines [36]. However, because the erythromycin zone diameter breakpoint is not available for Corynebacterium spp. in the EUCAST guidelines, the EUCAST interpretive criteria for Streptococcus pneumoniae was used. Accordingly, C. striatum strains showing an inhibition zone diameter of $\geq 22 \mathrm{~mm}$ were considered sensitive and $<19 \mathrm{~mm}$ were considered resistant. Similarly, the zone diameter breakpoint recommended by EUCAST for Viridans streptococci was used for cefotaxime. As such, C. striatum strains showing an inhibition zone diameter of $\geq 23 \mathrm{~mm}$ were considered susceptible and $<23 \mathrm{~mm}$ were considered resistant. Streptococcus pneumoniae ATCC 49619 was used for quality control [36]. The antibiotic susceptibility results of strains were recorded.

\subsection{Determination of the Clonal Relationship among C. striatum Strains}

The clonal relationship among C. striatum strains was determined using the arbitrarily primed polymerase chain reaction (AP-PCR) method. AP-PCR was carried out at the Inonu University Molecular Microbiology Laboratory as described previously [37]. The stock cultures of C. striatum strains were inoculated on 5\% sheep blood agar. After $24-48 \mathrm{~h}$ of incubation, pure bacterial cultures were obtained. Genomic DNA was isolated using the QIAsymphony total nucleic acid isolation kit. M13 primers (5'-GAGGGTGGCGGTTCT-3') were used for amplification under the following conditions: Initial denaturation for $5 \mathrm{~min}$ at $94^{\circ} \mathrm{C}$, followed by 40 cycles consisting of denaturation at $94{ }^{\circ} \mathrm{C}$ for $60 \mathrm{~s}$, annealing at $40{ }^{\circ} \mathrm{C}$ for $60 \mathrm{~s}$, and extension at $72{ }^{\circ} \mathrm{C}$ for $2 \mathrm{~min}$. The amplification products were separated by electrophoresis in a 1.5\% agarose gel with ethidium bromide for $1.5 \mathrm{~h}$ at $100 \mathrm{~V}$, and the band profiles were visualized using the Gel Logic 2200 imaging system (Kodak Co., Rochester, NY, USA) under ultraviolet light. The band profiles were analyzed using the Gel Compar version 6.6 software program (Applied Maths, Kortrijk, Belgium). The Dice Similarity Coefficient (DSC) was used in similarity calculations for band analysis, whereas the Unweighted Pairwise Grouping Mathematical 
Averaging (UPGMA) method was used for clustering analysis. The strains were identified as different genotypes if the DSC was $<95 \%$ and as the same genotype if it was $\geq 95 \%$. A dendrogram was created using the UPGMA clustering algorithm.

\subsection{Statistical Analysis}

The data were analyzed using the Minitab 17 (Minitab, Inc., PA, USA) statistical software program. Descriptive statistics were expressed as number (n), percentage (\%), and mean and standard deviation. The Kolmogorov-Smirnov test was performed to determine whether the variables were normally distributed. For the comparison of continuous variables, two-sample t-test was used. The Pearson's Chi-squared test or Fisher's Exact test was used for comparison of categorical variables if applicable. A probability $(P)$ value of $\leq 0.05$ was considered statistically significant in the $95 \%$ confidence interval.

\subsection{Ethical Approval}

This study was reviewed and approved by the Noninterventional Clinical Research Ethics Board of Karabuk University (Date: October 31, 2018; decision no: 10/11).

\section{Conclusions}

This study highlighted the increasingly frequent occurrence of C. striatum as an invasive infection/outbreak agent and the rising antibiotic resistance of strains. Although C. striatum is part of the normal human flora, it should not be overlooked when isolated from clinical specimens, as it may actually be a cause of infection. Therefore, the microbiologist and clinician should cooperate to reach a final decision. In the present study, C. striatum strains showed high-level resistance to most commonly used antibiotics. Therefore, based on our in-vitro antibiotic susceptibilities results, vancomycin, linezolid, and gentamicin can be selected for the empirical treatment of $C$. striatum infections. However, the antibiotic resistance profile of $C$. striatum should be monitored regularly, and empirical antibiotic treatment regimens should be revised. Although no single-clone C. striatum outbreak was detected in our hospital, polyclonal dissemination was detected and small clonal spreads were observed, indicating cross-contamination. Therefore, we should perform the effective infection control measures. In addition, compliance of HCWs to hand hygiene and cleaning of medical equipment and hospital surfaces with appropriate disinfectant should be monitored.

Supplementary Materials: The following are available online at http://www.mdpi.com/2076-0817/9/2/136/s1, Table S1: Distributions of C. striatum strains isolated from patients according to the age groups and gender; Table S2: Distributions of genotyped and sporadic C. striatum strains according to intensive care units and wards. Table S3: Distribution of genotyped and sporadic C. striatum strains according to the age groups.

Author Contributions: Conceptualization, N.A.; methodology, N.A., B.O.; resources, N.A.; B.O.; writing-original draft preparation, N.A.; review \& editing, B.O.; supervision, N.A., B.O.; project administration, N.A. All authors have read and agreed to the published version of the manuscript.

Funding: This research did not receive any external funding.

Acknowledgments: The authors would like to thank Enago for the English language review.

Conflicts of Interest: The authors declare no conflict of interest.

\section{References}

1. Kim, R.; Reboli, A.C. Other Coryneform Bacteria and Rhodococci. In Mandell, Douglas, and Bennett's Principles and Practice of Infectious Diseases, 8th ed.; Bennet, J.E., Dolin, R., Blaser, M.J., Eds.; Elsevier Inc.: Philadelphia, PA, USA, 2015; Volume 2, pp. 2373-2382.

2. Funke, G.; Bernard, K.A. Coryneform Gram-Positive Rods. In Manual of Clinical Microbiology, 11th ed.; Jorgensen, J.H., Pfaller, M.A., Carroll, K.C., Funke, G., Landry, M.L., Richter, S.S., Warnock, D.W., Eds.; American Society for Microbiology Press: Washington, DC, USA, 2015; Volume 1, pp. 474-503. 
3. Nhan, T.X.; Parienti, J.J.; Badiou, G.; Leclercq, R.; Cattoir, V. Microbiological investigation and clinical significance of Corynebacterium spp. in respiratory specimens. Diagn. Microbiol. Infect. Dis. 2012, 74, $236-241$. [CrossRef] [PubMed]

4. Bernard, K. The genus Corynebacterium and other medically relevant coryneform-like bacteria. J. Clin. Microbiol. 2012, 50, 3152-3158. [CrossRef] [PubMed]

5. Hahn, W.O.; Werth, B.J.; Butler-Wu, S.M.; Rakita, R.M. Multidrug-resistant Corynebacterium striatum associated with increased use of parenteral antimicrobial drugs. Emerg. Infect. Dis. 2016, 22, 1908-1914. [CrossRef] [PubMed]

6. Bowstead, T.T.; Santiago, S.M. Pleuropulmonary infection due to Corynebacterium striatum. Br. J. Dis. Chest 1980, 74, 198-200. [CrossRef]

7. Leonard, R.B.; Nowowiejski, D.J.; Warren, J.J.; Finn, D.J.; Coyle, M.B. Molecular evidence of person-to-person transmission of a pigmented strain of Corynebacterium striatum in intensive care units. J. Clin. Microbiol. 1994, 32, 164-169. [CrossRef]

8. Kammoun, M.M.; Regaieg, K.; Bahloul, M.; Ammar, R.; Bouaziz, M. Corynebacterium striatum meningitis. Med. Mal. Infect. 2016, 46, 454-456. [CrossRef]

9. Ramos, J.N.; Souza, C.; Faria, Y.V.; da Silva, E.C.; Veras, J.F.C.; Baio, P.V.P.; Seabra, S.H.; de Oliveira Moreira, L.; Hirata Júnior, R.; Mattos-Guaraldi, A.L.; et al. Bloodstream and catheter-related infections due to different clones of multidrug-resistant and biofilm producer Corynebacterium striatum. BMC Infect. Dis. 2019, 19, 672. [CrossRef]

10. Lee, J.Y.; Lee, S.H.; Kim, W.H. Three-valve Endocarditis Caused by Corynebacterium striatum. Korean Circ. J. 2018, 48, 861-862. [CrossRef]

11. Verma, R.; Kravitz, G. Corynebacterium striatum empyema and osteomyelitis in a patient with advanced rheumatoid arthritis. BMJ Case Rep. 2016. [CrossRef]

12. Renom, F.; Gomila, M.; Garau, M.; Gallegos, M.D.C.; Guerrero, D.; Lalucat, J.; Soriano, J.B. Respiratory infection by Corynebacterium striatum: Epidemiological and clinical determinants. New Microbes. New Infect. 2014, 2, 106-114. [CrossRef]

13. Verroken, A.; Bauraing, C.; Deplano, A.; Bogaerts, P.; Huang, D.; Wauters, G.; Glupczynski, Y. Epidemiological investigation of a nosocomial outbreak of multidrug-resistant Corynebacterium striatum at one Belgian university hospital. Clin. Microbiol. Infect. 2014, 20, 44-50. [CrossRef] [PubMed]

14. Qin, L.; Sakai, Y.; Bao, R.; Xie, H.; Masunaga, K.; Miura, M.; Hashimoto, K.; Tanamachi, C.; Hu, B.; Watanabe, H. Characteristics of multidrug-resistant Corynebacterium spp. isolated from blood cultures from hospitalized patients in Japan. Jpn. J. Infect. Dis. 2017, 70, 152-157. [CrossRef] [PubMed]

15. McMullen, A.R.; Anderson, N.; Wallace, M.A.; Shupe, A.; Burnham, C.A. When good bugs go bad: Epidemiology and antimicrobial resistance profiles of Corynebacterium striatum, an emerging multidrugresistant, opportunistic pathogen. Antimicrob. Agents Chemother. 2017, 61, e01111-e01117. [CrossRef] [PubMed]

16. Asgin, N. In-vitro Antibiotic Resistance Profile of Corynebacterium striatum Strains Isolated from Clinical Samples. ANKEM Derg. 2019, 33, 102-107.

17. Tran, T.T.; Jaijakul, S.; Lewis, C.T.; Diaz, L.; Panesso, D.; Kaplan, H.B.; Murray, B.E.; Wanger, A.; Arias, C.A. Native valve endocarditis caused by Corynebacterium striatum with heterogeneous high-level daptomycin resistance: Collateral damage from daptomycin therapy? Antimicrob. Agents Chemother. 2012, 56, 3461-3464. [CrossRef] [PubMed]

18. Ajmal, S.; Saleh, O.A.; Beam, E. Development of high-grade daptomycin resistance in a patient being treated for Corynebacterium striatum infection. Antimicrob. Agents Chemother. 2017, 61, e00705-e00717. [CrossRef]

19. Wang, Y.; Du, X.; Cui, J.; Wang, K.; Zhang, L.; Han, Y. Rapid transmission of multidrug-resistant Corynebacterium striatum among susceptible patients in a tertiary hospital in China. J. Infect. Dev. Ctries. 2016, 10, 1299-1305. [CrossRef]

20. Suh, J.W.; Ju, Y.; Lee, C.K.; Sohn, J.W.; Kim, M.J.; Yoon, Y.K. Molecular epidemiology and clinical significance of Corynebacterium striatum isolated from clinical specimens. Infect. Drug Resist. 2019, 4, 161-171. [CrossRef]

21. Martínez-Martínez, L.; Pascual, A.; Bernard, K.; Suárez, A.I. Antimicrobial susceptibility pattern of Corynebacterium striatum. Antimicrob. Agents Chemother. 1996, 40, 2671-2672. [CrossRef] 
22. Martínez-Martínez, L.; Suárez, A.I.; Winstanley, J.; Ortega, M.C.; Bernard, K. Phenotypic characteristics of 31 strains of Corynebacterium striatum isolated from clinical samples. J. Clin. Microbiol. 1995, 33, 2458-2461. [CrossRef]

23. Alibi, S.; Ferjani, A.; Boukadida, J.; Cano, M.E.; Fernández-Martínez, M.; Martínez-Martínez, L.; Navas, J. Occurrence of Corynebacterium striatum as an emerging antibiotic-resistant nosocomial pathogen in a Tunisian hospital. Sci. Rep. 2017, 7, 1-8. [CrossRef] [PubMed]

24. Wang, X.; Zhou, H.; Chen, D.; Du, P.; Lan, R.; Qiu, X.; Hou, X.; Liu, Z.; Sun, L.; Xu, S.; et al. Whole-Genome Sequencing Reveals a Prolonged and Persistent Intrahospital Transmission of Corynebacterium striatum, an Emerging Multidrug-Resistant Pathogen. J. Clin. Microbiol. 2019, 57, e00683-19. [CrossRef] [PubMed]

25. Shariff, M.; Aditi, A.; Beri, K. Corynebacterium striatum: An emerging respiratory pathogen. J. Infect. Dev. Ctries. 2018, 12, 581-586. [CrossRef]

26. Olender, A. Mechanisms of Antibiotic Resistance in Corynebacterium spp. Causing Infections in People. In Antibiotic Resistant Bacteria. A Continuous Challenge in the New Millennium, 1st ed.; Pana, M., Ed.; In Tech Open: London, UK, 2012; Volume 1, pp. 387-402.

27. Mumcuoglu, I.; Hazırolan, G.; Kurşun, Ş.; Aksu, N. Evaluation of the Corynebacterium striatum isolated with increasing frequency in one of the training and research hospital. Turk Hij. Den. Biyo. Derg. 2015, 72, 281-288. [CrossRef]

28. Neemuchwala, A.; Soares, D.; Ravirajan, V.; Marchand-Austin, A.; Kus, J.V.; Patel, S.N. In vitro antibiotic susceptibility pattern of non-diphtheriae Corynebacterium isolates in Ontario, Canada, from 2011 to 2016. Antimicrob. Agents Chemother. 2018, 62. [CrossRef]

29. Ortiz-Pérez, A.; Martín-De-Hijas, N.Z.; Esteban, J.; Fernández-Natal, M.I.; García-Cía, J.I.; Fernández-Roblas, R. High frequency of macrolide resistance mechanisms in clinical isolates of Corynebacterium species. Microb. Drug Resist. 2010, 16, 273-277. [CrossRef]

30. Barberis, C.M.; Sandoval, E.; Rodriguez, C.H.; Ramírez, M.S.; Famiglietti, A.; Almuzara, M.; Vay, C. Comparison between disk diffusion and agar dilution methods to determine in vitro susceptibility of Corynebacterium spp. clinical isolates and update of their susceptibility. J. Glob. Antimicrob. Resist. 2018, 14, 246-252. [CrossRef]

31. Campanila, F.; Carretto, E.; Barbarini, D.; Grigis, A.; Falcone, M.; Goglio, A.; Venditti, M.; Stefani, S. Clonal multidrug-resistant Corynebacterium striatum strains, Italy. Emerg. Infect. Dis. 2009, 15, 75-78. [CrossRef]

32. Fernandez-Cuenca, F. Applications of PCR techniques for molecular epidemiology of infectious diseases. Enferm. Infecc. Microbiol. Clin. 2004, 22, 355-360.

33. Baio, P.V.P.; Mota, H.F.; Freitas, A.D.; Gomes, D.L.; Ramos, J.N.; Sant'Anna, L.O.; Souza, M.C.; Camello, T.C.; Hirata, J.R.; Vieira, V.V.; et al. Clonal multidrug-resistant Corynebacterium striatum within a nosocomial environment Rio de Janeiro, Brazil. Mem. Inst. Oswaldo Cruz. 2013, 108, 23-29. [CrossRef]

34. Masroor, N.; Doll, M.; Stevens, M.; Bearman, G. Approaches to hand hygiene monitoring: From low to high technology approaches. Int. J. Infect. Dis. 2017, 65, 101-104. [CrossRef] [PubMed]

35. Godbout, E.J.; Masroor, N.; Doll, M.; Edmond, M.B.; Bearman, G.; Stevens, M.P. Bare below the elbows in an academic medical center. Am. J. Infect. Control. 2019, 4, 1030-1031. [CrossRef] [PubMed]

36. The European Committee on Antimicrobial Susceptibility Testing. Breakpoint Tables for Interpretation of MICs and Zone Diameters. Version 7.1, 2017. Available online: http://www.eucast.org/ast_of_bacteria/ previous_versions_of_documents/ (accessed on 10 April 2019).

37. Ayan, M.; Kuzucu, C.; Durmaz, R.; Aktas, E.; Cizmeci, Z. Analysis of three outbreaks due to Klebsiella species in a neonatal intensive care unit. Infect. Control Hosp. Epidemiol. 2003, 24, 495-500. [CrossRef] [PubMed]

(C) 2020 by the authors. Licensee MDPI, Basel, Switzerland. This article is an open access article distributed under the terms and conditions of the Creative Commons Attribution (CC BY) license (http://creativecommons.org/licenses/by/4.0/). 